\title{
If
}

HSOA Journal of Dentistry: Oral Health \& Cosmesis

\section{To Evaluate the Survival and Failure Rates of the Single Unit Full Coverage Crown Treatments}

Nankali Ali* and Unmesh Jyoti Nitin, orcid.org/0000-00017298-9311

Department of Adult Oral Health - Barts and the London, Queen Mary University, London, United Kingdom

\begin{abstract}
\section{Background and Aim}

Crown preparation and treatment is one of the most commonly performed procedures by dental practitioners. However, there have been very few studies, which have tried to focus on the aspect of crown survival. Although crown survival is multifactorial and complex, it is important to estimate the survival rate of dental crowns prepared by dental clinicians. It will help dental practitioners to answer patient's questions more confidently and would act as a means to communicate with them more effectively. Therefore, the aim of the clinical audit was to determine the causes of dental crowns survival as well as failure and the measures needed to be implemented to improve the survival rates.

\section{Materials and Methods}

The data collection was done at the Barts and The London Dental Hospital, London, United Kingdom. In this audit the collected data was formatted in a way to indicate aspects such as risk factors, mistakes and ways for improvement. However, the collected data for this project was entirely dependent on case notes of patients. Due to technical and other reasons, not all data was available which limited the sample size to 100 units.

\section{Results}

Results of this specific study shows that maxillary teeth have about 1.5 higher risk of being treated by crown than mandibular teeth. This times study also showed that survival rate at The Barts and The London Dental Hospital for crowns was $58 \%$ and the failure rate was $33 \%$. Observed failure rate in Barts Dental Hospital was about $13-14 \%$ higher than the acceptable rate of $20 \%$. In addition, 40-49 year aged group showed the maximum crown survival rate whereas the 60-69 year the least crown survival rate.

\section{Criteria for Survival and Failure of Coronal Coverage}

"Parameters used to describe the clinical performance of the single unit dental crowns were "Successful (Survival rate)" if the coronal coverage remained intact without any complications for
\end{abstract}

*Corresponding author: Nankali J Ali, Department of Adult Oral Health - Barts and the London, Queen Mary University, Turner Street, White Chapel, London, United Kingdom, Tel: +44 2078826353; E-mail: a.nankali@qmul.ac.uk

Citation: Ali N, Unmesh JN (2016) To Access Survival and Failure Rates of Single Unit Full Coverage Crown Treatments. J Dent Oral Health Cosmesis 1: 001.

Received: March 23, 2016; Accepted: August 17, 2016; Published: August 31, 2016 more than 5 years, any of the adverse events associated with an Fixed Dental Prosthesis (FDP) resulted in considered extraction of the abutment or crown removal in less than 5 years were considered "Unsuccessful (Failure rate)".

\section{Conclusion}

Further study is needed to be done in terms of evidences and generated results to be able to improve the standard of care, clarify whether practitioners are meeting currently available standards and there are any further requirements to set specific standards. In addition, the results of this study may bring more confidence for our dental practitioners who provide patient's needs. In conclusion, every practitioner should set criteria to improve crown survival rates. Clinicians should periodically compare their practice with the set standards, find out the failure reasons and re-audit- possibly against a modified standard.

Keywords: Audit; Crown longevity; Crown survival; Failure rates; Full coverage crowns; Survival rates

\section{Introduction and Background}

Innovations have helped to improve the quality of human life. Current era belongs to the technological advances and medical and dental field is no exception to these technological advances. It is changing drastically due to modern technological inventions with recent invention being the use of dental robot to drill the cavity on a tooth (GI Gadgets, 2016) [1]. Every aspect of the procedures as well as protocol can be measured, to determine the shortcomings in order improve quality of patient care, again big thanks to the technology.

Crown treatment requires certain skills and experience. It is one of the commonly performed treatment modalities in dentistry. Therefore, it is important to scrutinise its long term success rate to control and improve the possible outcomes in the future.

To achieve the aim and the objectives, an audit was considered as one of the appropriate methods for study because clinical audits can be undertaken either nationally or locally in trusts, hospitals, GP practices or any organisations where healthcare is provided. It involves many steps but the overall aim of an audit is to improve the patient care. Big organisations or institutions need to keep an eye on the quality of the work or the service being provided. Institutions spend huge sums of money on quality control because they know that improving it will earn them a higher profit [2]. This clinical audit was also a requirement of the institution. Audit can also serve as important tool to find out the potential factors impacting the successful treatment. Another factor is unlike any other field, success and failure of dental treatment depends on patient compliance and maintenance as well as providing best quality treatment by dental practitioner. Clarifying main related factors of the treatment failure would enable clinicians to work out appropriate answers to find out the underlying causes.

This study was done to determine the survival rate of the crown treatments performed in Barts and the London school of Medicine and Dentistry and to standardize the crown treatment.

\section{Aims and Objectives}

After considering the possible outcomes, the following aims and objectives were discussed and selected:

1) Set criteria and standards for crown longevity or survival. 
2) Compare current practice with the set standard.

3) Collect additional information to suggest reasons for failure

4) Suggest possible points that may impact the outcomes.

5) Re-audit- possibly against a modified standard. (Wales deanery, A Guide to undertake a clinical audit project [3]).

Terminology such as "successful treatment" can be ambiguous and depends on view of individual dental practitioner. Therefore, the term "Crown survival", was used, as it is the appropriate terminology.

\section{Importance of Crown Longevity Audit}

Crowns are categorised in band 3 in the United Kingdom (UK) National Health Service (NHS). Their quality and success or survival for long period of time is important to both the patient and the dentist. Crowns are technically challenging and involve clinician's skill and require a level of patient compliance. As far as the patient is concerned, the longevity of the crown is important in terms of money as well as health. Crown longevity is dependent on many patient factors such as the level of oral hygiene and the condition of the underlying tooth. Currently there are no standards and it would be possible to set criteria and standards for the minimum length of time most crowns can be expected to last set of guidelines [4].

\section{Materials and Methods}

This audit was conducted at Barts and The London School of Medicine and Dentistry, London, United Kingdom. Audit was approved with audit number " 6214 ". It took more than 3 months to collect the minimum required information to validate the study.

After conducting the literature search, two documents with relevant information were finalized. One was research paper published by Anusavice in year 2012 named "standardizing failure, success and survival decisions in clinical studies of ceramic and metal-ceramic fixed dental prosthesis" [5]. It was very helpful to get an idea about the guidelines of crown survival. Second set of guidelines were found in "Wales Deanery Cookbook". It played an important role in getting precise idea regarding crown audit and its guidelines. After comparing both set of the guidelines "Wales Deanery guidelines" were selected as a standard for data collection as it covered many similar areas such as success and failure rates of all types of crowns whereas, Anusavice paper considered only ceramic and Porcelain Fused to Metal (PFM) crown survival rate.

According to the selected guidelines, $80 \%$ of all crowns should last for at least 5 years. Therefore, acceptable failure rate was $20 \%$. This was selected as a gold standard and as a standard of good practice.

Method used to collect data of this study was retrospective analysis of patient record data from year 2003 to year 2009. The number of years were increased in order to get maximum records possible and to analyse long term survival and failure rates. Therefore, this audit can be termed as a retrospective audit

Initial search showed that there was not a specific database for patients who have undergone crown treatment at The Barts NHS Trust. Therefore, it was decided to access lab work entries of the patient who underwent fixed crown treatment from year 2003 to year 2009 as this data was stored in computerised system of Barts NHS trust and was available easily. From the obtained list of the lab work, patient's records were found out from the patient's case notes stored in the record room of Barts NHS trust. All the available list of patients were scrutinised thoroughly for five times in order to eliminate the factor of the human error and bias error. All case notes which were searched were manually arranged in alphabetical order according to surnames. Finding the patient's case notes according to the list was a very time consuming and laborious task.

Lab work list of the patients had 350 patients records from year 2003 to 2009 consisting of 565 units (crowns). To provide a fair study and simplify the work, mixed dentition cases with their complications were excluded from the study. After searching the case notes manually, $18 \%$ of the total patient list data (63 case notes) was available.

All of the available patients' case notes were analyzed which helped to achieve a sample size of 100 single unit crowns. Sample size was limited to 100 units as more data was not possible to obtain due to time constraint and technical difficulty.

To acquire the sample size, crown cementation date was noted (date/month/year). Then, all the pages of the case notes were scrutinized thoroughly to identify whether any retreatment was done to the placed crown and abutment tooth. Furthermore date of the last visit of patient to the hospital during last 10 years was indications for checking crown treatment information.

Crown failures were analyzed to determine the reasons of secondary visits such as re-cementation, fractures, extractions or any other issue and complication. Those crowns which didn't have any complaint in a span of 5 years from the cementation date were considered as successful treatments.

From all selected case notes 3 patients' records were not accepted because of the lack of information. The collected data were transferred to Microsoft Excel for interpretations and statistical analysis.

\section{Challenges}

A number of challenges were faced while conducting this study due to the fact that, this audit was retrospective audit and involved data from more than ten years.

- After comparing both set of the guidelines, One of the main challenges was to find out the relevant guidelines. Pubmed, Cochrane library, Scopus were the databases used to conduct literature search, search terms such as crown survival, full coverage crowns, survival rate, and crown longevity were used. Research paper published by Anusavice et al., in year 2012 named "standardizing failure, success and survival decisions in clinical studies of ceramic and metal-ceramic fixed dental prosthesis" was helpful to get an idea about the guidelines of crown survival [5]. Second set of guidelines were found in Wales Deanery Cookbook [3]. It helped in getting precise idea regarding crown audit and its guidelines.

- Some of the patients' records were not available and some had been destroyed, (10-20\% patients') due to long period of time span involved in the study (Year 2003- 2013).

- In addition, about 50 to $60 \%$ of patients notes mentioned in the lab work list had been moved to another storage room from where they were not accessible.

- Handwriting on the number of patient's case notes was not readable which led to some difficulties in interpretation of the data.

- Case note sheets were not numbered and arranged numerical order which led to unavailability of required data. 


\section{Results}

Microsoft Excel software was used to analyse the collected data. It led us to considering the following results and comparisons:

Within the limits of the conducted study,

- Maxillary teeth are at a higher risk of undergoing crown treatment as compared to their mandibular counterparts (Chart 1).

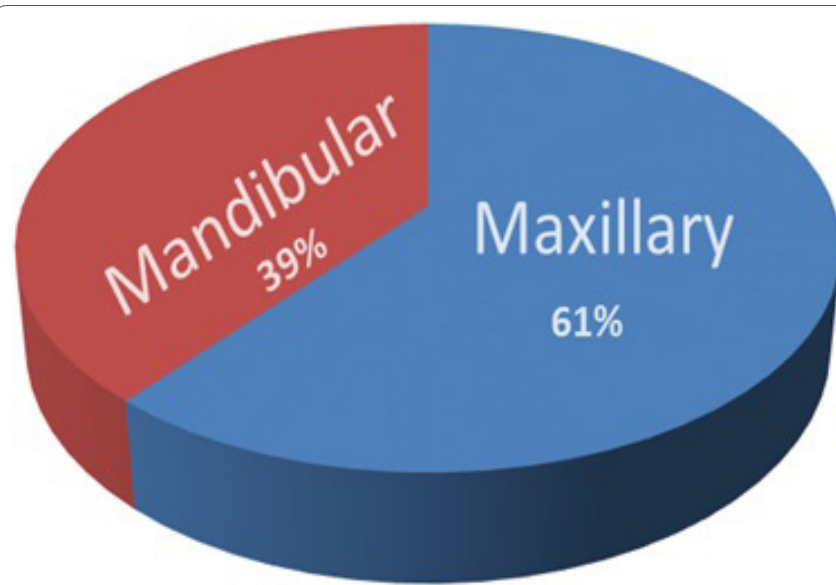

Chart 1: Ratio of maxillary to mandibular teeth. Blue area depicts the percentage of maxillary teeth and orange mandibular teeth.

- Calculation of successful and failed crown treatment rates in percentages was done which is illustrated in (Chart 2). After comparing to the acceptable failure rates as per the existing guidelines (Wales Deanery Guidelines), failure rates at the Barts and the London School of Medicine and Dentistry (Chart 3) was about 10$15 \%$ higher than acceptable rate. After analysing the failure rates (Chart 4), it was seen that the maximum number of crown treatment failures occurred during the first 5 years.

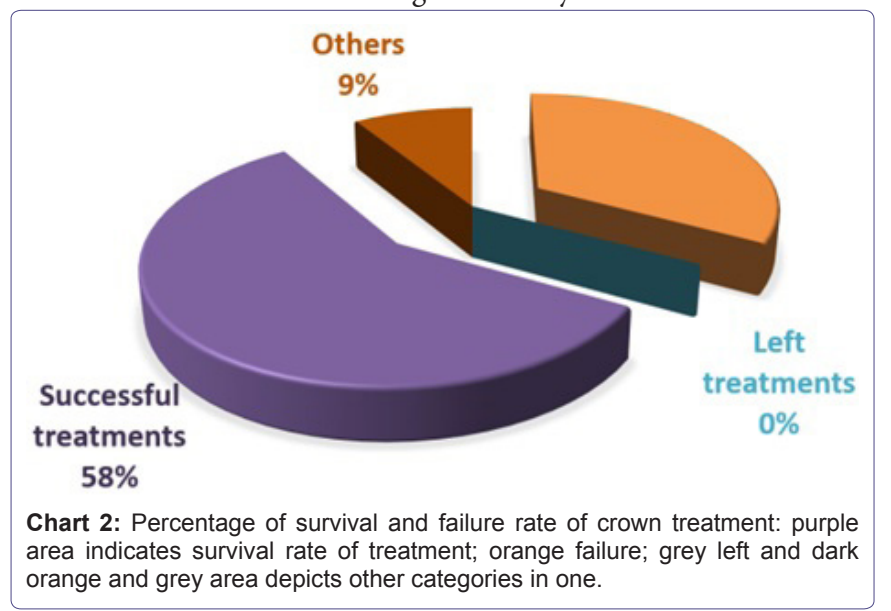

- Another finding of this study was there was not a single person who left the treatment in between.

- Statistically formatted table (Table 1 ) was created to determine the relation between maximum and least crown treatment survival rates. It was found out that, Age range of 40-49 years showed maximum crown survival rate $(\mathrm{p}=0.20)$, whereas $60-69$ years was the age range which showed the least survival rate $(\mathrm{p}=0.09)$.

- Percentages of each of the reasons of failures were calculated. According to the available information. (Chart 5). Failures were divided into following 7 groups.

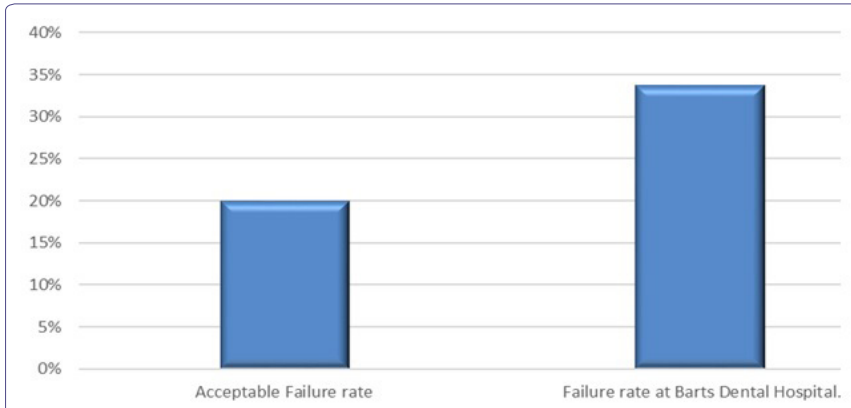

Chart 3: Comparison the acceptable failure rates and failure rate at Barts Dental Hospital and Wales Deanery Guidelines.

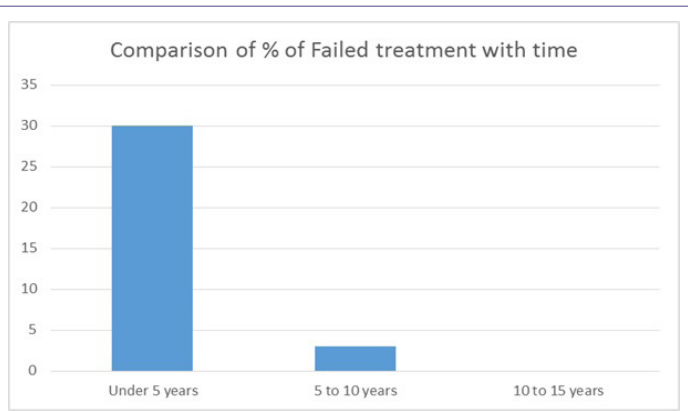

Chart 4: Comparison between rates of crown failure treatment and time period.

- Radiolucency below abutment

- Dislodgement of crown

- Extraction of abutment (due to mobility, fracture, pain, caries)

- History of repeated crown failures

- Fracture of Porcelain

- Removal of crown

- Recementation

It was found out that maximum failures were associated with the extraction of the abutment tooth due to the number of reasons such as pain due to caries, improper endodontic treatment, mobility due to periodontal problems followed by chipping or fracture of porcelain in patients with porcelain fused to metal or all ceramic crowns.

\section{Discussion}

It is important to emphasize that survival of dental treatment is a multifactorial process and complicated to evaluate. Yet, in this study an attempt has been made to evaluate those factors.

The study shows that the success rate is related to a number of factors which may divided into two substantial groups: patient factors and non-patient factors.

Patient related factors considered in this study included,

- Para-functional habits

- Maintenance of good oral hygiene

- Occlusion

- Non-patient related factors included

- Preparation (Taper, reduction, height, Ferrule effect)

- Luting cement (Quality, quantity) 


\begin{tabular}{|c|c|c|c|c|c|c|c|c|c|c|c|c|}
\hline \multirow{3}{*}{ Age } & \multicolumn{6}{|c|}{ Observation } & \multicolumn{6}{|c|}{ Probability of the Observation } \\
\hline & \multirow{2}{*}{$S$} & \multicolumn{3}{|c|}{$\mathrm{F}$} & \multirow{2}{*}{ LT } & \multirow{2}{*}{ Others } & \multirow{2}{*}{$S$} & \multicolumn{3}{|c|}{$\mathrm{F}$} & \multirow{2}{*}{ LT } & \multirow{2}{*}{ Others } \\
\hline & & $\mathrm{T} \leq 5$ & $5<\mathrm{T} \leq 10$ & $10<T$ & & & & $\mathrm{~T} \leq 5$ & $5<\mathrm{T} \leq 10$ & $10<T$ & & \\
\hline $20-29$ & 7 & 6 & 0 & 0 & 0 & 0 & 0.09 & 0.08 & 0.00 & 0.00 & 0.00 & 0.00 \\
\hline $30-39$ & 6 & 6 & 1 & 0 & 0 & 4 & 0.08 & 0.08 & 0.01 & 0.00 & 0.00 & 0.05 \\
\hline $40-49$ & 15 & 4 & 0 & 0 & 0 & 4 & 0.20 & 0.05 & 0.00 & 0.00 & 0.00 & 0.05 \\
\hline $50-59$ & 13 & 6 & 0 & 0 & 0 & 0 & 0.17 & 0.08 & 0.00 & 0.00 & 0.00 & 0.00 \\
\hline $60-69$ & 6 & 7 & 1 & 0 & 0 & 1 & 0.08 & 0.09 & 0.01 & 0.00 & 0.00 & 0.01 \\
\hline $70-79$ & 11 & 1 & 1 & 0 & 0 & 0 & 0.15 & 0.01 & 0.01 & 0.00 & 0.00 & 0.00 \\
\hline
\end{tabular}

Table 1: Statistical analyses of survival and failure rates according to the age groups. S - Successful treatment; LT - Left treatment; F - Failed treatment.

\section{$\square$ The age range with maximum crown survival rate}

\section{$\square$ The age range with least crown survival rate}

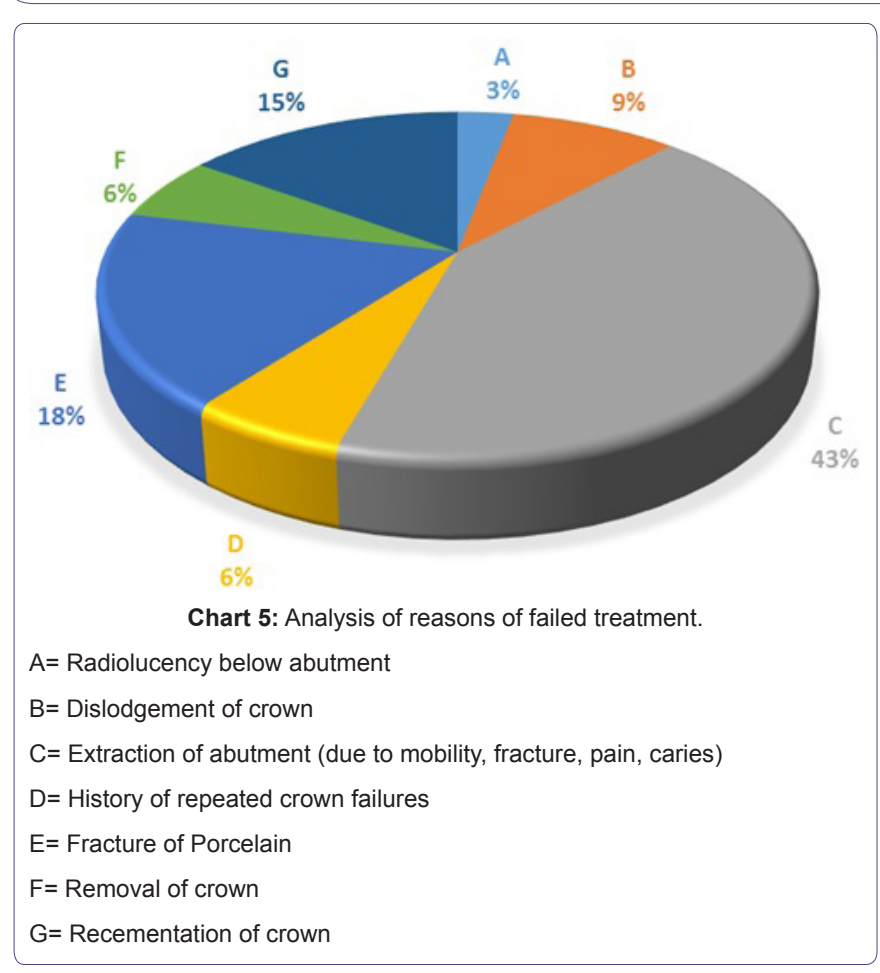

- Impression (Techniques, materials)

- Crown fabrication and manufacturing defects ( Laboratory factors, materials)

These factors are already discussed, studied and published separately by a number of authors and this audit once again demonstrated a strong relation between them and crown survival rates. These correlations address thoughtful considerations of the factors such as para-functional habits, oral hygiene and occlusion while developing the treatment plan by dental practitioners to reduce the failure rates of the crown treatment.

Crown preparations should be also monitored to increase the survival rate. Similarly, Luting cement issues need to be resolved.

It is important to mention that patient should be educated and motivated adequately so that he can maintain optimum level of oral hygiene. From the analysed factors for failure of crowns in results section, it was thought important to discuss factors such as quality of endodontic treatment. According to one study, "incidence of periapical lesions on radiographs of crowned teeth was low during 25 years" observation in root-filled teeth with high quality endodontic treatment and coronal coverage" [6]. It is important to crown the teeth with large caries with vital pulp and to follow recommended protocols while providing dental treatment as the study proves that, "Proper designing and morphology of dowel and core have a similar survival rate as crowned teeth with a vital pulp. A high proportion of crowned teeth with a vital pulp remain free from signs and symptoms of pulpal deterioration over 25 years" [6].

One study focussed on tooth survival of endontically treated teeth without corornal coverage showed that "overall survival rates of endodontically treated molars without crowns at 1,2 , and 5 years were $96 \%, 88 \%$ and $36 \%$, respectively. With greater amounts of coronal tooth structure remaining, the survival probability increased. Molar teeth with maximum tooth structure remaining after endodontic treatment had better survival rate after 5 years" [7]. One more study showed that "the risk involved in losing the endodontically treated posterior teeth to fracture if not supported by full cast crown is too high to take. To help reinforce the cusps of pulpless teeth weakened by tooth structure removal, the authors recommend the use of crown that encompasses the cusps to withstand the occlusal forces of everyday mastication. Clinically over a period of 25 years that root canal treated posterior teeth, irrespective of the amount of tooth structure lost either by caries or access cavity preparation, mostly fracture if not protected by full cast crowns [8]. Therefore, these studies reflect the fact that it is important to have coronal coverage after completion of endodontic treatment for long time survival of clinical crown and abutment.

In terms of materials used to restore the tooth after endodontic treatment, this study showed that, "post endodontic restorations with direct composite had a better survival rate than conventional amalgam and reinforced zinc oxide and eugenol with polymethacrylate restorations. The amounts of remaining tooth structure and types of restorative material have significant association with the longevity of endodontically treated molars without crown coverage" [7].

It was tried to validate the audit by formatting the collected data in an appropriate way for studying and analyzing the outcomes. However, for better monitoring the quality and quantity of treatment it was suggested in an external/internal audit meeting at Barts and The London Dental Institute to follow up the notes, sequences and update discharged patients and repeat the audit cycle.

In conclusion, it is important to do an audit on crown survival which would give an idea about previous and on-going dental 
practice. This will equip the practitioner with confidence in the treatment they provide in addition to providing benefit to the patients.

\section{Limitations of the Study}

- In this study, analysis of only single unit crowns were done. Multiple unit bridges were not selected due to time factor and availability of records.

- Data collection of the patients in this project was entirely depended to case notes. As mentioned before, due to old data and other reasons many records could be found. It limited data collection to 100 . If more records were available, more data could have been collected. As it is known that higher the sample size, more distinguishable and unbiased is the results.

- Patients whose data was not available were contacted by telephone but as many of the patients had moved their houses and had changed the phone numbers, this was not successful.

- As case notes were searched manually, there is a chance of human error.

\section{Suggestions and Future of the Project}

\section{Need of improvement in survival rates of crowns}

As can be seen from results and interpretation of this audit project, crown survival rate after 5 years was found out to be $58 \%$ which is less by $22 \%$ than expected. Therefore, there is definitely a need to review treatment protocol and analyse dentist related reasons.

\section{Need to device an updated system of filing data for future analysis}

As mentioned before, the biggest problem in data collection was improper handwriting. Some case notes were not organized properly. Therefore, there should be some system to monitor these problems so that in future data collection will be easier. Suggestion after analyzing all the problems regarding data collection would be to create a new program for easy data collection in future, in which practitioners would enter the data after finishing each treatment or cementation of crowns. This coded data could be applied to some devices like iPad that are used currently and in future.

\section{Follow up of the discharged patients}

Following up of the discharged patients can play important role in tracking the crown survival. This can be done by creating software for auto contact (text-mail) with discharged patients in a certain time interval. There is no need to appoint a separate person for it.

\section{Implement a monitoring system for handwriting in patient notes and date arrangements}

Practically, it is impossible to keep track on each and every practitioner for handwriting and it is sensitive issue as it may hurt some practitioners' ego. Hence, best solution for this problem would be to inform practitioners by gentle reminder or display message regarding this matter.

\section{Study the reasons behind higher percentage of maxillary} teeth treated with crown compared to mandibular

As it was seen in interpretation of analyzed data, within limits of this study and audited sample, maxillary teeth have $22 \%$ higher risk of being treated by crown as compared to mandibular teeth. More data is needed to verify this observation and understand possible reasons.

Find and analyse reasons why certain age group of people have distinguishable positive and negative survival rates

As seen from the interpretations of statistical table 3.1, within limits of this study, 40-49 year was the age range which showed the most crown survival rate whereas 60-69 years was the age range which showed the least crown survival rate. It is very important to find correlation between crown survival rate and age and other involved factors.

\section{Conclusion}

Further studies are needed to build evidence so as to improve the standard of care in the UK. This will help to clarify whether practitioners are meeting currently available standards and if there are any further requirements to set specific standards.

The results of this study may help to highlight to dental practitioners factors that are important in crown survival rates for our dental practitioners who provide patient's needs. In conclusion, every practitioner should set criteria for herself or himself to improve crown longevity and survival rates. Clinicians should periodically compare their practice with the set standard, analyse the failure reasons and re-audit- possibly against a modified standard.

\section{References}

1. GI Gadgets (2016) Portable dental machine can clean tooth cavity with accuracy.

2. Crombie JK, Davies HTO, Abraham SCS, Florey C (1993) The Audit Handbook, Chichester: John Wiley and Sons Ltd, New Jersey, USA.

3. Wales deanery (2006) A guide to undertake a clinical audit project, Cookbook, Clinical Audit Peer Review, School of Postgraduate Medical and Dental Education, Cardiff University, Cardiff, United Kingdom.

4. Croydon K (2015) A guide to undertake clinical audit project, Wales deanery, Cardiff, UK.

5. Anusavice KJ (2012) Standardizing failure, success, and survival decisions in clinical studies of ceramic and metal-ceramicfixed dental prostheses. Dent Mater 28:102-111.

6. Valderhaug J, Jokstad A, Ambjørnsen E, Norheim PW (1997) Assessment of the periapical and clinical status of crowned teeth over 25 years. J Dent 25: 97-105.

7. Nagasiri R, Chitmongkolsuk S (2005) Long-term survival of endodontically treated molars without crown coverage: A retrospective cohort study. J Prosthet Dent 93: 164-170.

8. Tikku A, Chandra A, Bharti R (2010) Are full cast crowns mandatory after endodontic treatment in posterior teeth? J Conserv Dent 13: 246-248. 